\title{
The annual reports of Local Research Ethics Committees
}

\author{
Claire Gilbert Foster, Tim Marshall and Peter Moodie King's College, London, and Birmingham University
}

\begin{abstract}
Each Local Research Ethics Committee (LREC) is expected to produce an annual report for its establishing authority. Reports from 145 LRECs were examined with regard to (a) whether the committees were working within the terms of the most recent guidelines from the Department of Health and (b) observations on the role of LRECs with particular reference to accountability.

Most LRECs had produced a report, although their length varied greatly. Most reports showed how seriously the committee took its task. Most committees met many of the guidelines; for example, almost all had two or more lay-members. The guideline most frequently not met was that committees should have no more than 12 members.

Many committees review very large numbers of projects (maximum 351). Approximately two-thirds provide details in the annual report of individual project titles, their author and the committee decision; all reports should contain this information. Although it may in fact happen more generally, only 23 per cent of the reports referred to any form of monitoring of the eventual outcome of the research.

$A$ significant issue to arise from the reports is the extent to which the framework for the operation of LRECs has been confused by the development of the purchaser-provider split. The paper concludes with suggestions for remedying the situation.
\end{abstract}

\section{Introduction}

There have been several accounts of the functioning of Local Research Ethics Committees. An early and influential report was by Nicholson (1), which dealt specifically with the issue of research on children. Other reports have described practice in the UK (2) and Scotland (3), the experiences of lay-members of LRECs (4), and of researchers trying to pilot the protocol for a multi-centre study through 30 different LRECs (5). Meanwhile both Gilbert et al (6) and Neuberger (7) have undertaken reviews of LREC

\section{Key words}

Research ethics committees; annual reports. practice, the first by post and the second by observation and interview. One of the conclusions to be drawn from these reports was that LRECs were characterised by their variability rather than by any clear consistency of practice.

The Department of Health or its predecessors have at various times produced guidelines concerning the establishment, responsibilities and practice of LRECs. Most recently, the department issued an updated set of guidelines (8), setting out expectations for the establishment and functioning of LRECs, for implementation by District Health Authorities (DHAs) by March 1992. For the firs time it was made clear that each LREC shoule produce an annual report for its establishin authority, and that the report should be available for public inspection. It was through this mechanism that LRECs were to have some degree of accountability for their work.

\section{Aims and objectives}

This paper is the report of a study undertaken for the Centre of Medical Law and Ethics at King's College, London. There were three objectives: (i) to assess whether the Department of Health guidelines concerning the existence of LRECs and their publication of annual reports were being met; (ii) to assess, on the basis of information published in the annual reports, the level of adherence to the guidelines relating to the constitution and operation of LRECs, and (iii) to assess the information from the annual reports in the context of current debate about the role of LRECs, with particular reference to issues of accountability.

\section{Design of the study}

The Department of Health (DoH) guidelines suggest that there should normally be one LREC for every District Health Authority. The guidelines envisage that there might occasionally be two (but no more than two) and suggest that the circumstances in which this might be appropriate could be 'where there is a particularly high burden of work, 
perhaps originating from two research centres' (9). Of other criteria for the establishment of more than one committee - for example, by geography, or type of research - there is no mention. This issue was not particularly contentious or problematic at the time the guidelines were issued, but recent months have been a time of great flux for health authorities, with increases in the number of National Health Service (NHS) trusts and the merger of many authorities. These changes might be expected to have had a major effect on the work of the LRECs involved, but a recent circular indicated that the jurisdictional bias of LRECs should remain unaltered (10). Accordingly, we based this survey upon District Health Authorities, and obtained an up-to-date list of those authorities in membership of the National Association of Health Authorities and Trusts (NAHAT 143).

A letter and brief questionnaire were sent, addressed to the chair of the LREC in each authority, requesting a copy of the most recent annual report of the LREC. Some were returned from different authorities where mergers had recently taken place. Because of the recent organisational changes mentioned above, we felt that it would be prudent to ask the chairmen whether they knew of the existence of any other LREC within the district and if so to let us have contact addresses. In a few instances the 'second' committees were informed directly by the secretariat of the primary committee and material was sent directly to us. To others we wrote. A followup mailing or telephone call went to those who failed to respond to the first enquiry. We are grateful to all those chairmen and administrative staff who helped us by responding to our requests.

Even before letters were sent to the 'second' committees it had become clear that the model propounded by the department of one or possibly two LRECs within a district did not accord with current practice. We discovered that in some districts there were three or even more LRECs. In a few districts there was no district-wide LREC. In these districts one of several different models was in operation: the 'extra' committees reflected decisions that ethical review should be institution-based (a committee relating to a single hospital), function-based (a committee dealing with nursing, student or other projects), geographically based (perhaps reproducing boundaries that preceded a merger of health authorities), or trust-based (where the committee was the responsibility of a National Health Service trust).

For these reasons it is quite inappropriate to base our report solely on single district-based LRECs, and in the analysis which follows, unless stated otherwise, all LRECs from which we obtained information are included (145). No separate enquiries have been made of Family Health Service Authorities (FHSAs) with regard to their arrangements for the ethical review of research.

\section{Results}

\section{(I) LRECS AND THEIR ANNUAL REPORTS}

The guidelines require that each year the LREC should submit a report to the DHA. It should give the names of committee members, the numbers of meetings held and should include a list of proposals considered by the committee, including whether they were approved, approved after amendment, rejected or withdrawn (8).

One hundred and forty-three health authority LRECs were circulated in the first instance and we were informed of a further 52 LRECs. Of the 143 districts, annual reports were received from 110 , there was an indication from 25 that no report had been produced, and despite reminders, no response from 5 . Three committees no longer existed following administrative changes at health authority level. Including all of the other ways of acquiring reports described above, 145 annual reports were received in total. The jurisdictional basis of these LRECs is shown in Table 1. It should be noted that several of the 'district-wide' committees from whom we received reports are no longer district-wide, following mergers of health authorities, whilst others no longer exist.

The 'annual' reports covered a variety of periods, ranging from under 6 months to over 18 months. However, the vast majority (119 (82 per cent)) were for a 12-month period. The length of the reports also varied greatly, from one side of A4 paper to substantial bound documents of 40 or more pages.

Eighty-nine reports (61 per cent) gave the full title of each protocol considered, together with a clear indication of the outcome of each application. A substantial minority (55 (38 per cent)) did not provide these details, offering only totals for the number of projects received, and those accepted or rejected. One committee advised us that the whole of their report was confidential, and several others that the details (title, etc) of each project and the decision about it could not be released.

\section{(II) MEMBERSHIP OF LRECS}

The guidelines lay down a number of requirements for the membership of LRECs. The committee

\section{Table 1}

\section{The jurisdictional basis of LRECs}

District-wide

Geographical part of district

NHS trust-based

Non-trust, institution-based (for example, single hospital)

Function-based (for example, nursing) Other

TOTAL 


\begin{tabular}{|c|c|}
\hline \multicolumn{2}{|c|}{$\begin{array}{c}\text { Table } 2 \\
\text { The size of LRECs }\end{array}$} \\
\hline Number of members & Number of committees \\
\hline$<8$ & 4 \\
\hline 8 & 9 \\
\hline 9 & 17 \\
\hline 10 & 21 \\
\hline 11 & 24 \\
\hline 12 & 18 \\
\hline$>12^{\star}$ & 34 \\
\hline `Maximum size: 19. & \\
\hline
\end{tabular}

should have between eight and twelve members, who should be drawn from both sexes and from a wide range of age groups. They should include hospital medical staff, nursing staff, general practitioners and two or more lay persons. The meaning of 'lay persons' is not defined, although the guidelines appear to envisage at least two kinds: 'at least one lay member should be unconnected professionally with health care and be neither an employee nor adviser of any NHS body' (11).

We cannot be certain from the annual reports whether many committees satisfy all of the guideline requirements. No annual report gave members' ages, and many were inconclusive as to the gender of at least some members, although 118 (81 per cent) gave the names of members as required by the guidelines. One of the most frequent grounds on which LRECs fail to meet these requirements is the size of the committee. Table 2 gives the number of members on the committees where this information was provided, and shows that approximately one quarter of these contained more than the maximum proposed in the guidelines.

The guidelines specify that the individual affiliation of members (for example, GP, nurse, etc) should be stated in the report. This information was only available in 123 ( 85 per cent) of the reports. The guidelines also require each committee to have either a lay chairman or vice-chairman. The reports gave a clear indication that this requirement was met in 86 committees ( 59 per cent), of which 30 had a lay chairman.

Almost all committees met the requirement to have at least two lay-members. No committee appeared to be without a lay-member, and only seven had a single lay-member. Most commonly committees had two lay-members (62 (43 per cent)), whilst 54 ( 37 per cent) had three or more. Forty-seven committees ( 32 per cent) could be identified as having a clergyman amongst the membership, and 31 (21 per cent) had a lawyer. These figures, particularly that for lawyers, must be taken as minima, since lay-members' occupations were not always given. There was no indication in the reports that any religious representative was of $\frac{1}{Q}$ non-Christian faith.

(III) LRECS AT WORK

The annual reports show that the amount of $\vec{F}$ business conducted by individual LRECs varies enormously. Table 3 makes use only of those reports (119) which cover a 12-month period. The first two columns show the number of meetings held by the 104 LRECs providing this information. There is clearly very great variation in the number of $\%$ meetings held; but this variation in workload is seen $\vec{\circ}$ even more clearly in the numbers of protocols reviewed by these committees. The second half of Table 3 shows that some of the 114 committees providing this information handle a very large number of protocols indeed in a twelve-month period.

Many reports indicated that there was some concern about the workload of members of the com- is mittee, with 35 referring to this issue; for example, a meeting of one committee lasted over $6 \frac{1}{2}$ hours. 을 Although several alluded to an increase in the number of protocols submitted, very few gave any quantitative data to illustrate their concern. In a small number of cases the report suggested there was a very significant shortfall in the degree of administrative or secretarial support available. The guideg lines require (para 2.1) that the DHA should provide administrative support; it seems that a few districts are not providing this support effectively.

The guidelines suggest (para 2.14) that LRECs should require immediate notification of unusual or unexpected results which raise questions about the safety of the research, and also suggest that reports from investigators on successes or difficulties in recruiting subjects may provide the committee with useful feedback on the acceptability of the project to patients and volunteers. A very few reports indicate that the occasional project was terminated because of unexpected side-effects or difficulty with recruitment, but this seems to be a rare occurrence. More broadly, $\delta$ 23 per cent of the LRECs do refer to the existence of some form of routine monitoring of protocols which has been approved by the committee. This monitoring normally takes the form of requiring six- or twelve-monthly reports from the investigator on the progress or conclusion of the research.

One aspect of the working practices of LRECs on $N$ which the guidelines are silent is the question of $N$ whether commercial organisations sponsoring a protocol should be expected to contribute to the costs of the ethical review process. The Department $\Phi$ of Health has recently stated that it is not, in $\stackrel{\odot}{+}$ principle, against LRECs charging (in its letter 7 accompanying an EU Directive on Medical Devices) (12). The majority of annual reports do not refer to $\Omega$ this issue, but it is clear that several LRECs do make $\stackrel{\mathbb{Q}}{\varrho}$ a charge for consideration of some protocols, and $\bar{Q}$ that these charges vary considerably, from $£ 25$ to $£ 250$ per protocol. 
Table 3

The activity of ethics committees

\begin{tabular}{|c|c|c|c|}
\hline Number of meetings & Number of committees & Number of protocols & Number of committees \\
\hline$<6$ & 26 & $<25^{\star}$ & 19 \\
\hline 6 & 24 & $25-<50$ & 41 \\
\hline 7-9 & 17 & $50-<100$ & 29 \\
\hline 10,11 & 23 & $100-<200$ & 13 \\
\hline$\geqslant 12$ & 14 & $\geqslant 200 \dagger$ & 12 \\
\hline
\end{tabular}

The annual reports suggest that there is considerable variation in the style of working of the committees. For example, there was firm evidence from the reports that 28 of the committees routinely invite those who had submitted protocols to attend the meeting at which they were discussed. However, additional comments suggested that for several committees this would be the case only where some potential difficulty had been apprehended before the meeting.

\section{(IV) ISSUES OF IMPORTANCE TO LRECS}

Many chairmen in their annual report take the opportunity to comment on issues which are of concern to them. Perhaps the most significant set of comments relate to the uncertainties caused for some committees by the organisational changes in the NHS. The merger of health authorities has inevitably caused some difficulties and confusion, and the development of NHS trusts has also raised questions about the remit of some committees. The implications of the purchaser-provider split within the NHS was the basis of several comments; this issue is pursued in the discussion section of this paper.

The question of how to provide for the ethical review of multi-centre research has long been an issue of concern $(5,13)$. The guidelines start from the proposition that each LREC is free to arrive at its own decision in all cases, but then suggest voluntary arrangements whereby one LREC is nominated to consider the issue on behalf of them all (14). A few of the annual reports indicate that steps are being taken in this direction, although it is clear that the arrangements normally result only in an advisory opinion which would not bind the individual LRECs. In a more general sense, 26 reports (18 per cent) made some reference to the issue of multi-centre research. The most frequently expressed view was that, however much time might be saved by having some element of central or regional overview of such protocols, local circumstances were so important that they should never be ignored when it came to implementing research in a specific setting.

\section{Discussion}

One overriding impression from reading the annual reports is how seriously LRECs take their task. There is nothing to indicate that committees which may fail to adhere to the guidelines in terms of their composition or manner of working in any way compromise their concern for the interests of either researchers or subjects. Indeed, there is an argument that the guidelines themselves are unhelpful in that in some respects they are too prescriptive (why should they assume that a committee of more than a dozen members is always to be avoided?), and in other respects inadequately detailed (no mention of charging commercial organisations for the work involved).

\section{(I) ACCOUNTABILITY}

Many of the points which arise from a consideration of the reports relate to the issues of accountability and the need to reassure patients, and the public, that medical research is subject to effective ethical review. The guidelines make it clear (para 1.1) that the decision on whether research goes ahead must come from the NHS body (trust, HDA, or FHSA) under whose auspices the research would take place. This perspective has recently been re-stated (10). However, there is very little in the LRECs' reports to indicate that in practice the effective decision is taken by anyone other than the LREC itself. It is an oddity of the structure envisaged by the guidelines that the only detailed public review of research proposals comes from the annual report of what is in theory an advisory body, rather than from the body which is formally responsible for the final decision.

Nevertheless, it remains important that the annual reports of LRECs should contain all the information which would serve to reassure the public. In particular, and as a minimum, there seems to be no reason for not giving the outcome of each individual application considered by the committee. And, of course, we can see no defence for the view of the one committee which told us that its entire report was confidential.

The principle of accountability suggests that the names of committee members should be known, and 
the guidelines (para 2.16) require this. There may be other points of view. Some of the committees excluding members' names from their reports did so expressly because they did not wish the names to be known to sponsors of research. However, even if names of individuals are not given, we suggest that an indication of the post or occupation of the members should be given, since this could give reassurance that the committee comprised a broad range of experience.

A few reports indicated that a junior doctor was a member of the committee. Since it will often be junior medical staff who enrol patients into research studies this seems a desirable extension of the more typical range of membership. We also believe that it would be sensible to extend the guidelines' suggestion of a lay-member unconnected professionally with health care from one to at least two of the membership of the committee. Other 'lay'members, such as hospital chaplains, may have a valuable contribution to make, but the objective of creating an effective, independent element in the review process suggests that they might serve in addition to two truly lay people.

It is good to find that many committees make efforts, as described earlier, to follow up the research which they approve. However, the reports generally give little indication of how successful these efforts are. None of the reports makes any reference to the active monitoring of research whilst it is in progress. Active monitoring would go beyond current practice in order to ensure that research is carried out as originally approved. At present, many committees spend a good deal of their time considering, for example, how subjects' consent should be obtained, but little is done to ensure that patients are always approached and informed in precisely the agreed manner, let alone that the research itself is conducted in accordance with the approved protocol.

\section{(II) RESOURCES}

We have referred earlier to the need for all LRECs to be properly supported for all the necessary requirements of their task; the onus for ensuring that this happens must be on the DHA which is responsible for the committee. This obligation will principally comprise the provision of administrative support. However, the need for training of LREC members must also receive attention. Several committees referred to various aspects of training in the reports, although no report suggested the existence of a plan for its provision. Various initiatives are under way in this area: we hope that the issue of training for LRECs and their individual members will receive sustained attention in the future.

\section{(III) DISTRICT-BASED LRECS IN A TRUST-BASED} SERVICE?

Perhaps the single most significant issue which arises from these annual reports is the impact of NHS re-organisation on the system of ethical review. The $\frac{2}{\mathbb{D}}$ guidelines were issued before NHS trusts becamem the main basis for the delivery of health care, though $\overrightarrow{\overline{\overline{ }}}$. the principles of the new organisational arrange-

ments were already established (15).
It is clear that the proposal that a district LREC should be used to provide ethical research advice to? all NHS bodies within the district (para 2.1) has $\frac{\bar{c}}{\bar{s}}$ resulted in considerable confusion, for both districts $\vec{\sigma}$ and trusts. In the internal market, where income for hospitals has become of considerable significance, there is a new set of direct pressures affecting the $\overrightarrow{-}$ carrying out of sponsored research. If a major objective of an effective system of ethical review is tow maintain and increase public confidence that all decisions are being made for laudable reasons, then there is a strong argument for maintaining the basisi of review of hospital-based research in an external $\overrightarrow{+}$ body, namely the district.

On the other hand, it could be argued that the $\vec{f}$ purchasing authority is not necessarily the most은 appropriate body to approve or refuse applications to carry out research. It seems out of place with the remainder of the system that a body advising a majoro purchaser of services should concern itself with what $\stackrel{\text { क }}{\rightarrow}$ are in effect the minutiae of the clinical affairs of a $\overrightarrow{0}$ provider unit. In some circumstances it might seegnc invidious to researchers in one institution to hawe their work approved, or not, after detailed review gy clinicians from another trust who sit on the distriet LREC.

\section{Confusing picture}

It is clear that the guidelines did not adequately envisage the issues which would arise for LRECs out of the recent changes in the NHS. We cannot overemphasize the confusing picture which has developed following the merger of many district: authorities, and the development of the purchaser- 3 . provider split. There are districts where a merger has $\delta$ resulted in the creation of a new LREC, but where $₹$ the old LRECs are still functioning, never havingo been told to disband. In some districts a committee that was essentially a hospital-based organisation (though performing a district-wide function), with administrative support provided by the district, has. found that support withdrawn when the hospital N achieved trust status, and the chairman unable to discover if his or her committee is still supposed too exist or to whom it should report. In other districtso such committees have continued explicitly as trust- $\bar{\Phi}$ based committees, though their relation to newly? established district-wide committees in the same 0 district remains uncertain. In yet other districts, the committee acts as an umbrella focal point, receiving

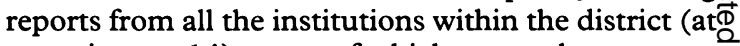
a maximum, 14), some of which currently are trusts and some of which are not, but each of which has its own REC. It is fair to point out that confusion is far 
from universal. In many merged districts clear decisions have been taken to merge, or sometimes not to merge, old LRECs, and ethical review seems to be proceeding coherently with everyone clear about reporting and accountability.

Nevertheless, it is important that ground rules should now be considered for the situation which has developed and that any new proposals should take account of the diversity described above. In our view it is desirable that, as the 1991 guidelines recommended, in most situations the basic template should remain the single district-wide LREC. However, the existence of some degree of flexibility to take account of local circumstances cannot be avoided. The minimum requirement must be that a DHA should be made aware of the existence of all LRECs operating within its geographical area, and that the district and the Department of Health should maintain a list of all such LRECs. The most important substantive point is that each DHA should authorise and approve the creation, constitution and membership of every LREC within its district, should provide the requisite administrative support, and should receive an annual report from each of them. Where an LREC presently exists without the formal approval of the DHA, this approval should be sought. Without such a requirement it will be more difficult for those engaged in medical research to convince the public of the independence and accountability of the process of ethical review.

Claire Gilbert Foster, BA, is Research Fellow at the Centre of Medical Law and Ethics, King's College, London, and is running the Research Ethics Committee Project. In this capacity she has produced the Manual for Research Ethics Committees, which is updated annually, and is running training courses for members of research ethic committees. She is lay vice-chairman of Central Oxford Research Ethics Committee. Tim Marshall, MBE, MSc, CStat, is Senior Lecturer and Acting Head of the Department of Public Health and Epidemiology, University of Birmingham. His main teaching responsibilities are in Epidemiology and Public Health for undergraduate medical students, and he is used widely as a Consultant Statistician by local clinicians. He has been a member of South Birmingham
Research Ethics Committee for many years, and is currently Vice-Chairman. Peter Moodie, BA, DipCrim (Cantab), is a Senior Lecturer and Assistant Head of the School of Law in the University of Birmingham. He is Course Leader for courses in Criminal Law and Criminology, but has also for many years contributed to courses on Medical Ethics and Law for both medical and law students. He has for several years been a member of a Local Research Ethics Committee in Birmingham.

\section{References}

(1) Nicholson R H, ed. Medical research in children: ethics, law and practice. Oxford: Oxford University Press, 1986.

(2) Wells F O, Griffin J P. Ethics committees for clinical research: experience in the UK. Drugs 1989; 37: 229-232.

(3) Thompson I E, French K, Melia K M, Boyd K M, Templeton A A, Potter B. Research ethical committees in Scotland. British medical journal 1981; 282: 718-720.

(4) West Birmingham CHC. Ethical committees. Birmingham: West Birmingham Community Health Council, 1989.

(5) Harries U J, Fentem P H, Tuxworth W, Hoinville GW. Local research ethics committees: widely differing responses to a national survey protocol. fournal of the Royal College of Physicians 1994; 28: 150-154.

(6) Gilbert C, Fulford K W M, Parker C. Diversity in the practice of district ethics committees. British medical journal 1989; 299: 1437-1439.

(7) Neuberger J. Ethics and health care: the role of research ethics committees in the United Kingdom. London: King's Fund Institute, 1992.

(8) Department of Health. Local research ethics committees. London: Department of Health, 1991.

(9) See reference (8): para 2.3.

(10) Department of Health. Research ethics committees and the National Health Service reforms. Health trends 1993; 25, 1: 2.

(11) See reference (8): para 2.7.

(12) Department of Health. EC directives and local research ethics committees. Letter to LREC chairmen, 1993: Dec 23.

(13) Meade $T$ W. The trouble with ethics committees. Fournal of the Royal College of Physicians 1994; 28, 2: 102-104.

(14) See reference (8): para 2.18.

(15) Department of Health. National Health Service and Community Care Act. London: Her Majesty's Stationery Office (HMSO), 1990. 